\title{
Correction to: A Normative Reference vs. Baseline Testing Compromise for ImPACT: The CARE Consortium Multiple Variable Prediction (CARE-MVP) Norms
}

\author{
Breton M. Asken ${ }^{1,2}$ (1) . Zachary M. Houck ${ }^{2}$. Julianne D. Schmidt ${ }^{3} \cdot$ Russell M. Bauer $^{2}$. Steven P. Broglio ${ }^{4}$. \\ Michael A. McCrea ${ }^{5} \cdot$ Thomas W. McAllister $^{6}$. James R. Clugston ${ }^{7}$. Care Consortium Investigators
}

Published online: 3 March 2020

(c) Springer Nature Switzerland AG 2020

\section{Correction to: Sports Medicine \\ https://doi.org/10.1007/s40279-020-01263-2}

Unfortunately, in the published article the symbol "\%ile" has incorrectly been published as "\%o". We have now corrected this in all the occurrences.

The original article has been corrected.

The original article can be found online at https://doi.org/10.1007/ s40279-020-01263-2.

Breton M. Asken

breton.asken@ucsf.edu

1 Department of Psychiatry and Human Behavior, Alpert Medical School of Brown University, Providence, USA

2 Department of Clinical and Health Psychology, University of Florida, HSC PO Box 100165, Gainesville, FL 32610, USA

3 Department of Kinesiology, University of Georgia, Athens, GA, USA

4 Michigan Concussion Center, School of Kinesiology, University of Michigan, Ann Arbor, MI, USA

5 Departments of Neurosurgery and Neurology, Medical College of Wisconsin, Milwaukee, WI, USA

6 Department of Psychiatry, Indiana University School of Medicine, Indianapolis, IN, USA

7 Department of Community Health and Family Medicine, University Athletic Association, University of Florida, Gainesville, FL, USA 